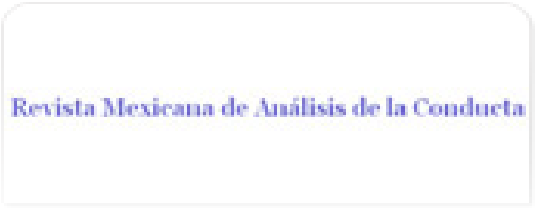

Revista Mexicana de Análisis de la Conducta ISSN: 0185-4534

editora@rmac-mx.org

Sociedad Mexicana de Análisis de la Conducta México

ALVARADO, ANGÉLICA; VILA, JAVIER; STREMPLER-RUBIO, ENEIDA; LÓPEZ-ROMERO, LUIS J.

Aprendizaje espacial y recuperación espontánea en humanos

Revista Mexicana de Análisis de la Conducta, vol. 37, núm. 2, 2011, pp. 139-153

Sociedad Mexicana de Análisis de la Conducta

Guadalajara, México

Disponible en: http://www.redalyc.org/articulo.oa?id=59319255009

- Cómo citar el artículo

- Número completo

- Más información del artículo

- Página de la revista en redalyc.org

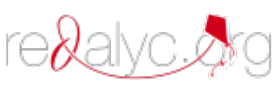

Sistema de Información Científica

Red de Revistas Científicas de América Latina, el Caribe, España y Portugal Proyecto académico sin fines de lucro, desarrollado bajo la iniciativa de acceso abierto 
REVISTA MEXICANA DE ANÁLISIS DE LA CONDUCTA MEXICAN JOURNAL OF BEHAVIOR ANALYSIS

DOI: $10.5514 /$ rmac.v37.i2.26144
2011

$37,139-153$
NUM $2(8-11 / 11-11)$

\title{
APRENDIZAJE ESPACIAL Y RECUPERACIÓN ESPONTÁNEA EN HUMANOS
}

\author{
SPATIAL LEARNING AND SPONTANEOUS RECOVERY IN HUMANS
}

\section{ANGÉLICA ALVARADO, JAVIER VILA, ENEIDA STREMPLER-RUBIO Y LUIS J. LÓPEZ-ROMERO}

\author{
F. E. S. Iztacala, Universidad Nacional Autónoma de México,
}

Tlalnepantla, Estado de México, México

\begin{abstract}
Resumen
El objetivo del presente trabajo fue demostrar el efecto de recuperación espontánea en humanos empleando una tarea de nado virtual similar a la piscina de Morris. Se presentan dos experimentos con una tarea de aprendizaje espacial de nado virtual (VSL Nesplora $($ ). En el primer experimento los participantes aprendieron a localizar la plataforma de salida que se ubicó entre los puntos de referencia que formaron los objetos A y B en 8 ensayos. En el experimento dos, a dos grupos se les presentó la tarea espacial del experimento anterior, durante la primera fase la plataforma se ubicó entre los objetos $A$ y $B$, mientras que durante la segunda entre $C$ y $D$, ambas fases constaron de 12 ensayos. Se realizó una prueba con los cuatro objetos presentes y sin la plataforma, un grupo a las $0 \mathrm{~h}$ y al segundo $24 \mathrm{~h}$ después. Los resultados mostraron que cuando la prueba se realiza a las $0 \mathrm{~h}$ la búsqueda de la plataforma se realiza en base a los puntos de referencia $C$ y $D$ pero a las $24 \mathrm{~h}$ se observa una elección intermedia entre A-B y C-D. El modelo experimental presentado es contrastado con los estudios de aprendizaje espacial en animales que han observado efectos asociativos.

\footnotetext{
Esta investigación fue apoyada con financiamiento de los proyectos DGAPA-UNAM (IN302910). La correspondencia relacionada al presente artículo debe ser dirigida a Angélica Serena Alvarado García, División de Investigación y Posgrado, Facultad de Estudios Superiores Iztacala, UNAM, AP 314, Tlalnepantla, Edo. Mex, 54096, MÉXICO. email: aserena77@hotmail.com. En el presente trabajo Javier Vila colaboró en la elaboración de la introducción y discusión, así como en los diseños experimentales. Eneida Strempler colaboró en el diseño y aplicación de la tarea experimental, así como en la recolección de datos y su representación. Luis Jesús López Romero colaboró en el diseño y aplicación de la tarea, así como en el análisis de datos. Y Angélica Alvarado participo en la escritura general del manuscrito, elaboración de diseños experimentales, así como en la representación y análisis de datos.
} 
Palabras clave: Aprendizaje espacial, tarea de nado virtual, recuperación espontánea, humanos.

\begin{abstract}
The aim of present study was to demonstrate spontaneous recovery effect in humans using a swimming virtual task analog to Morris pool. Two experiments with a task of spatial learning of swim virtually (VSL Nesplora (C) are presented. In the first experiment the participants learned to locate in 8 trials the exit platform that was located between the points of reference that formed the objects A and B. In Experiment 2, the spatial task of the previous experiment was presented to two groups, but during the first phase the platform was located between objects A and B, whereas during the second one it was located between C and D; both phases consisted of 12 trials. A test was performed with the four objects present and without the platform, a group at $0 \mathrm{~h}$ and the second one $24 \mathrm{~h}$ later. The results showed that when the test was made at 0 , platform searching is based on landmarks $C$ and $D$, but when the test was made at $24 \mathrm{~h}$ an intermediate choice between landmarks $A B$ and $C D$ was observed. The experimental model presented is contrasted with the studies of spatial learning in animals where associative effects have been observed.
\end{abstract}

Key words: Spatial learning, swimming virtual task, spontaneous recovery, humans.

El aprendizaje y la memoria espacial se relacionan con la capacidad de adquirir y retener asociaciones de las características del ambiente, lo que permite al organismo desenvolverse en el espacio, por lo que tanto, para animales como para humanos es de vital importancia conocer y ubicar objetos en el ambiente ya que de ese conocimiento depende su supervivencia. Así aquellas especies capaces de encontrar alimento, de protegerse de eventos hostiles e incluso de encontrar rutas alternativas para llegar a una meta tendrán una mejor adaptación a su medio (Shettlewoorth, 2010).

Durante las décadas de los cuarentas y los cincuentas la investigación sobre aprendizaje espacial se centró en indagar qué y cómo aprendían las ratas a resolver un laberinto. Tolman (1948) propuso la idea de "mapa cognitivo", como una representación cognitiva de la situación experimental, de tal manera que las ratas resolvían el laberinto asociando lugares de éste con la recompensa, es decir, un aprendizaje de tipo estímulo-estímulo (E-E). Por otro lado, Hull (1943) explicó el aprendizaje espacial de acuerdo a una asociación estímulo-respuesta (E-R), en donde la rata asociaba un lugar del laberinto con una respuesta que llevaba a una recompensa. Ambas formas de explicar el aprendizaje espacial recurren a un mecanismo asociativo, ya que la observación del aprendizaje E-E y E-R dependerá de la naturaleza de las claves disponibles en la situación experimental (Restle, 1957).

Aproximaciones posteriores (O'Keefe, \& Nadel, 1978), han propuesto que el término "mapa cognitivo" hace referencia a un proceso más complejo que el solo 
hecho de formar asociaciones simples. La propuesta de estos autores, supone la existencia de un campo cartográfico del espacio en dónde se encuentran las claves espaciales, por lo que es una representación del espacio y constituye el marco de referencia para poder orientarse. Lo que supone dos estrategias de aprendizaje que emplea el organismo: la estrategia de aprendizaje guiado que implica aproximarse al objetivo por medio de señales específicas como el color, la forma, el olor o la textura presentes en el ambiente y la estrategia de aprendizaje localizado que implica formar un mapa cognitivo del ambiente, que se actualiza constantemente, para encontrar la localización específica del objetivo.

Una técnica actual para el estudio del aprendizaje espacial ha sido el laberinto de agua de Morris (1981) en el cual una rata es colocada en una piscina circular de agua translúcida que contiene una plataforma de salida escondida por debajo del nivel del agua, que el sujeto deberá encontrar en el menor tiempo posible. Para ello los animales pueden orientarse empleando como referencia varias claves u objetos colocados en puntos determinados en el exterior de la piscina. En este aprendizaje de escape, las ratas disminuyen la latencia con la que encuentran la plataforma de salida a medida que transcurren los ensayos de entrenamiento. En los últimos años se han desarrollado versiones virtuales de esta tarea a fin de ser empleadas en investigación en aprendizaje espacial humano, y los resultados obtenidos han sido consistentes con los reportados en roedores (Sandstrom, Kauffman, \& Huettel, 1998; Livingstone, \& Skelton, 2007).

Recientemente empleando esta técnica, en animales y humanos algunos teóricos del aprendizaje asociativo (Chamizo, 2002, 2003; Pearce, 2009) han considerado que en el aprendizaje espacial opera un mecanismo asociativo, es decir, que existe un mapa cognitivo que permite que los organismos encuentren un objetivo mediante referencias espaciales. Dicho mapa cognitivo se forma por medio de las representaciones de las claves espaciales y el objetivo. De esta forma se propone que las estrategias de aprendizaje espacial propuestas por O'Keefe y Nadel (1978), tienen en realidad un mecanismo asociativo, ya que las claves de referencia interactúan mutuamente de manera similar al condicionamiento de un compuesto luz-tono, tal y como ocurre en el efecto de ensombrecimiento (Pavlov, 1927).

Así, Chamizo, Azar-Casanova y Artigas (2003) observaron el efecto de ensombrecimiento en humanos, empleando una preparación virtual de la piscina de Morris. Los participantes fueron entrenados a encontrar una plataforma invisible o visible, de acuerdo al grupo, ubicada entre dos señales colocadas entre los cuatro puntos cardinales por fuera de la piscina. Durante la prueba la plataforma fue removida. La salida para el inicio de cada ensayo variaba entre los 4 puntos cardinales de manera aleatoria. Los resultados demostraron varios puntos: primero, que los participantes durante la prueba pasaron más tiempo en el cuadrante donde fue ubicada la plataforma durante el entrenamiento, marcada con las dos señales ya fueran cercanas o lejanas en relación a la plataforma; segundo, que se observó el efecto de ensombrecimiento cuando estuvieron presentes las cuatro señales durante la prueba y cuando 
en el entrenamiento la plataforma fue visible. Los autores concluyen que con dichos resultados se observa un claro efecto de ensombrecimiento debido a que la fuerza asociativa de cada señal disminuye cuando hay más elementos entre los cuales se puede repartir dicha fuerza asociativa, tal y como ocurre entre el tono y la luz en un procedimiento de condicionamiento. Estos resultados sugieren que los mecanismos subyacentes al aprendizaje espacial son similares a los del condicionamiento simple (Chamizo, 2002; 2003).

Por otro lado, recientemente se ha demostrado que los efectos de recuperación de una respuesta extinguida pueden ser observados en el aprendizaje espacial con animales, en dónde al igual que en el condicionamiento pavloviano, después de la extinción se observa un resurgimiento de la respuesta condicionada después de un intervalo de retención o cambio de contexto físico. Así el efecto de recuperación espontánea fue observado por Prados, Maintenga y Sansa (2003; Experimento 1) quienes entrenaron dos grupos de ratas en una piscina de Morris para encontrar una plataforma oculta por debajo del agua, la cual era opaca debido a la mezcla con pintura. Después de $24 \mathrm{~h}$ de la fase de adquisición, se realizó una fase de extinción, en dónde la plataforma fue removida de su sitio. Después de 0 o 96 h del término de la fase de extinción, las ratas fueron probadas. El grupo de ratas que se probo a las $0 \mathrm{~h}$ después de la fase de extinción distribuyo el tiempo del ensayo de prueba buscando en los 4 cuadrantes en los que fue dividida la piscina, de manera que su búsqueda estuvo ubicada en niveles del azar, mientras que el grupo que fue probado a las 96 h, paso más tiempo buscando la plataforma en el cuadrante en donde se ubicó la plataforma durante la fase de adquisición. Demostrando así la recuperación de una respuesta extinguida como función del paso del tiempo.

Por lo tanto, el objetivo del presente trabajo es por un lado, replicar los resultados obtenidos en el aprendizaje espacial en humanos empleando una tarea de nado virtual que simula informáticamente la preparación de nado en piscina de Morris y que es equivalente a la piscina original empleada en ratas (VSL Nesplora $\odot$ ). Y por otro, demostrar si la recuperación espontánea ocurre en la tarea de nado virtual con participantes humanos, de manera similar a como ocurre en otras preparaciónes de aprendizaje con humanos (Alvarado, Jara, Vila, \& Rosas; 2006; López-Romero, García-Barraza, \& Vila, 2010). Para lo cual se esperaría que los resultados de los participantes sean similares a los presentados por los sujetos de Prados, et al., (2003). La importancia de la réplica sistemática del efecto de recuperación espontánea en humanos en el dominio espacial radica, por un lado en el aumento de la generalidad del efecto, y por otro en la posibilidad de suponer mecanismos asociativos básicos comunes entre humanos y animales en el aprendizaje espacial.

\section{Método General}

Participantes

Participaron de manera voluntaria e informada de acuerdo a los requerimientos 
éticos para la investigación con humanos de la FES Iztacala, 24 estudiantes de licenciatura, hombres y mujeres, de la carrera de Psicología de la Facultad de Estudios Superiores Iztacala UNAM y del Centro Interdisciplinario de Ciencias de la Salud Sto. Tomás del Instituto Politécnico Nacional, con edades entre los 18 y 25 años, sin experiencia con la tarea.

\section{Situación experimental}

Los experimentos se llevaron a cabo en un cubículo individual de $1.5 \times 1.5 \mathrm{~m}$, en las instalaciones de la Facultad de Estudios Superiores y en un salón de $3 \times 4$ m, en las instalaciones del Centro Interdisciplinarios de Ciencias de la Salud St. Tomás del Instituto Politécnico Nacional. Ambos espacios equipados con una mesa y una silla y una computadora compatible IBM con un monitor de $38 \mathrm{~cm}$. El procedimiento se implementó con el software comercial para el estudio del aprendizaje espacial Nesplora VSL 1.0 ( ) (virtual spatial learning), y la participación fue de manera individual. Los estímulos empleados como marcadores o guías fueron objetos prediseñados en el programa informático: A: pelota, B: cono, C: esfera y D: cubo.

\section{Tarea Experimental}

Se les solicitó a los participantes que "nadaran" en un ambiente que simulaba una piscina virtual de forma circular para localizar una plataforma invisible. La plataforma se ubicó entre dos de los objetos guías que se encontraban por fuera de la piscina ( $\mathrm{A}$ y B) en cada uno de los cuatro puntos cardinales (norte sur, este y oeste), la posición de los objetos se contrabalanceó de manera que los participantes al iniciar el ensayo visualizaran un objeto guía diferente en el punto cardinal norte. Todos los elementos de la tarea se presentaron en escala de grises. Los participantes usaron las teclas de navegación del teclado para desplazarse por la piscina y la barra espaciadora para avanzar al ensayo o pantalla siguiente. Cada ensayo estuvo programado para durar 60s, el cual terminaba al cumplirse el tiempo o si los participantes encontraban la plataforma. Cuando los participantes encontraban la plataforma aparecía la pantalla de retroalimentación que decía: "Objetivo alcanzado", sí se cumplían los 60 s, del ensayo aparecía la pantalla de retroalimentación que decía: "Objetivo no alcanzado". Las instrucciones fueron presentadas por escrito, además de ser leídas por el experimentador, quién salió de la habitación en cuanto el participante manifestó entender en qué consistía la tarea. En la Figura 1 se muestra como ejemplo las pantallas que los participantes observaron durante la tarea.

Como variables dependientes se consideraron, la latencia en encontrar la plataforma invisible durante bloques de dos ensayos, así como el tiempo que cada participante permanecía en cada uno de los cuadrantes de la piscina circular. Las cuales fueron registradas automáticamente por el programa Nesplora VSL 1.0 @ 

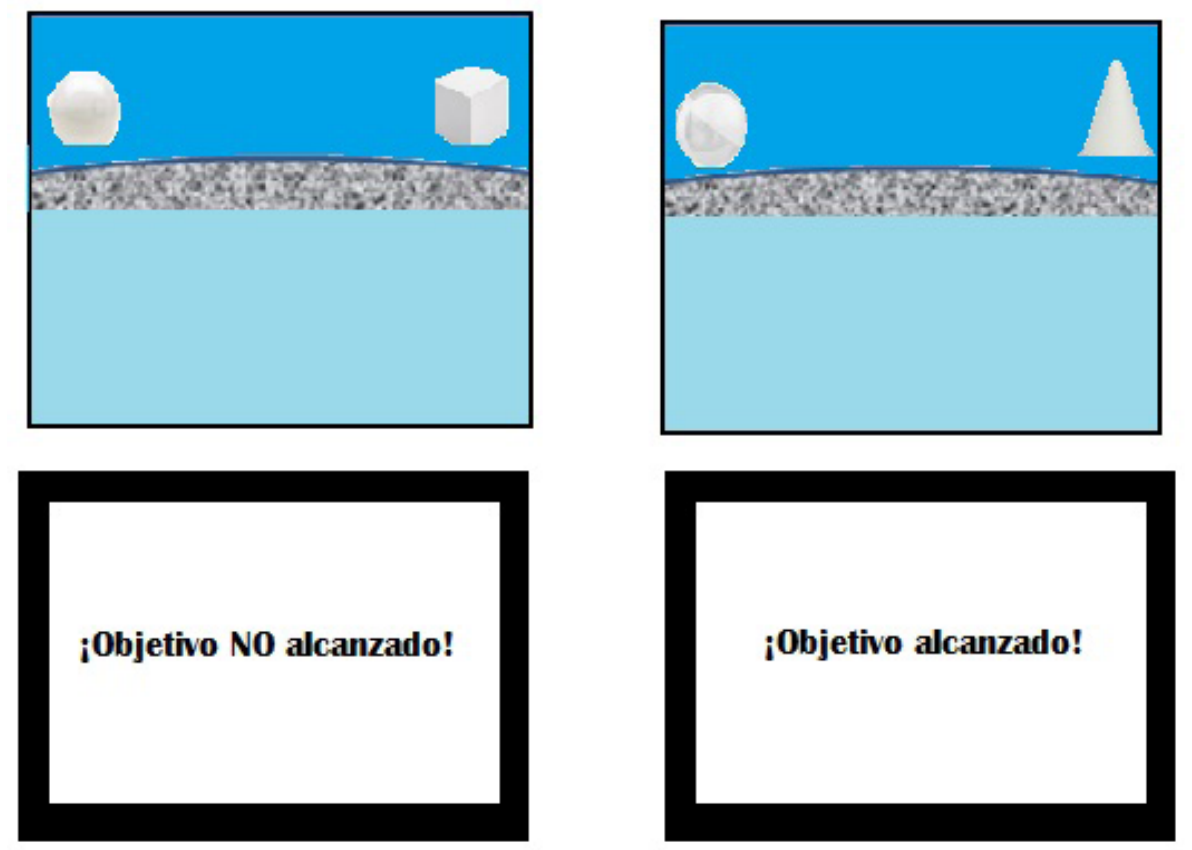

Figura 1. La figura muestra la tarea de nado virtual y la pantalla de retroalimentación presentada a los participantes durante los ensayos de entrenamiento.

\section{Experimento 1}

Este experimento se diseñó para replicar el aprendizaje espacial en humanos, empleando la tarea de nado virtual y búsqueda de al plataforma meta con humanos. Se espera encontrar que los participantes al igual que los animales no humanos en piscina de Morris disminuyan la latencia para encontrar la plataforma de salida a medida que transcurran los ensayos de entrenamiento. Tal y como ha ocurrido en la literatura en estudios que han empleado la piscina virtual de Morris (Sandstrom, et. al., 1998; Livingstone, \& Skelton, 2007)

Método

\section{Participantes}

Participaron de manera voluntaria e informada ocho estudiantes de licenciatura, 5 hombres y 3 mujeres, con las características descritas en el método general. 
Situación experimental y materiales

El experimento se llevó a cabo bajo las condiciones y con los materiales descritos en el método general.

\section{Tarea Experimental}

Se empleó la tarea descrita en el método general, con la plataforma invisible ubicada entre los objetos guías A (pelota) y B (cono).

\section{Procedimiento}

Al entrar al cuarto experimental se les pidió a todos los participantes que se sentaran frente al monitor de la computadora y que leyeran las instrucciones. Después el experimentador leyó las instrucciones en voz alta y preguntó al participante si existían dudas sobre lo que tenía que hacer. Si existían dudas se resolvían de manera verbal, si no se daba inicio a la tarea, dejando solo al participante en el cubículo. Los participantes debían presionar la barra espaciadora para dar inicio a la tarea.

Los participantes realizaron un ensayo de familiarización con las flechas de navegación del teclado, durante este ensayo la plataforma fue visible y no se encontraban los objetos que fungieron como guías por fuera de la piscina. Posteriormente se presentaron los 8 ensayos de entrenamiento con la plataforma invisible.

En el primer ensayo de entrenamiento, en el punto cardinal norte apareció el objeto $\mathrm{D}$ (cubo) como primer objeto guía visible para el participante, en el segundo ensayo fue el objeto C (esfera), en el tercero apareció el objeto A (pelota) y en el cuarto ensayo el objeto $\mathrm{B}$ (cono); en los últimos cuatro ensayos el primer objeto guía fueron las mismas figuras, pero en orden inverso (BACD). El arreglo de los ensayos mencionado con anterioridad, pretendió adaptar las condiciones del programa Nesplora a la tarea de nado de ratas en piscina de Morris.

\section{Resultados}

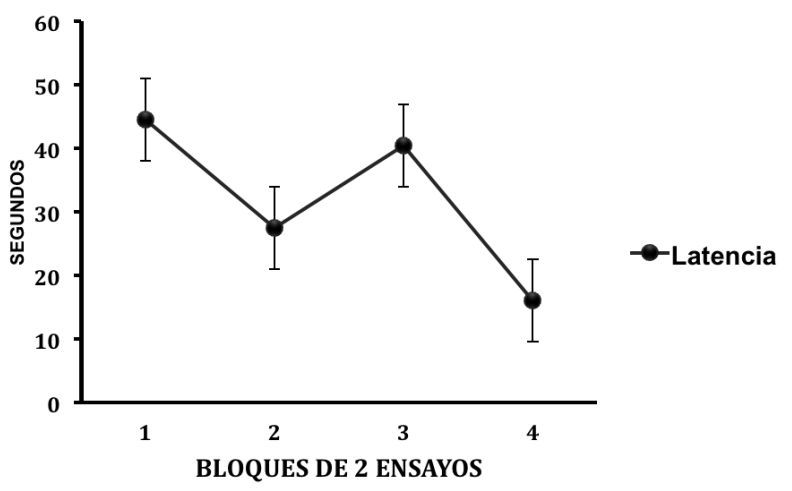

Figura 2. Se presentan las latencias en segundos en bloques de 2 ensayos de la respuesta de encontrar la plataforma. 
Los resultados se muestran en la Figura 2, y se puede observar que la latencia promedio disminuyó durante los ensayos de entrenamiento lo que sugiere que todos los participantes aprendieron a encontrar la plataforma invisible de salida. Estos resultados muestran que los participantes aprendieron a ubicar la plataforma empleando los objetos guía colocados en el exterior de la piscina.

Un análisis de varianza para medidas repetidas de una vía de los bloques de ensayos de los participantes $(\mathrm{F}(1,3)=4.14942, \mathrm{p}<0.05)$ mostró que los participantes tardaron menos tiempo en encontrar la plataforma invisible conforme pasaron los ensayos de entrenamiento, ya que las latencias en el primer bloque de 2 ensayos fueron de $90 \mathrm{~s}$, mientras que en el bloque 4, fueron cercanas a los $15 \mathrm{~s}$, aún cuando en el bloque 3 se observa un incremento debido a que fue durante éste bloque que dio inicio la fase de interferencia y la plataforma fue retirada del cuadrante $A B$ y colocada en el cuadrante CD. Lo anterior significa que los participantes después de ocho ensayos de entrenamiento aprendieron a ubicar la plataforma de acuerdo a los objetos que se encontraron por fuera de la piscina.

Los presentes resultados son similares a los obtenidos por Morris (1981) en ratas y coherentes con los resultados encontrados con humanos en una piscina de nado virtual (Sandstrom, et. al., 1998; Livingstone, \& Skelton, 2007). De esta manera la navegación virtual en humanos es una herramienta con la que se puede estudiar el aprendizaje espacial de manera similar a la tarea de nado en piscina de Morris con ratas.

\section{Experimento 2}

La recuperación espontánea (RE) se refiere a la reaparición de una respuesta condicionada después de un intervalo de tiempo ó retención, misma que fue aprendida en una primera fase de entrenamiento, seguido de una fase de extinción o de interferencia (Pavlov, 1927; Rescorla; 2004). Actualmente es un efecto robusto que ha sido observado en casi todas la preparaciones de condicionamiento.

El fenómeno de recuperación espontánea en aprendizaje espacial ha sido observado empleando un diseño de extinción en tareas de navegación en piscina de Morris con ratas después de un intervalo de retención de $96 \mathrm{~h}$ (Prados, et al., 2003). Sin embargo al presente, no existe una demostración con humanos en aprendizaje espacial, por lo que el presente estudio busca demostrar la recuperación espontánea empleando una preparación de nado virtual con humanos con un diseño de interferencia. En la tarea los participantes, en una primera fase aprenden a localizar una plataforma invisible en una piscina circular en el cuadrante que forman dos objetos guías que se ubican por fuera de la piscina (A-B) y en una segunda fase la plataforma es cambiada al cuadrante C-B. Se espera que los participantes después de un intervalo de retención busquen la plataforma en el cuadrante A-B que aprendieron inicialmente. 
Aprendizaje espacial y recuperación espontánea

Método

\begin{abstract}
Participantes
Participaron de manera informada y voluntaria estudiantes universitarios, 6 mujeres y 10 hombres de entre 18 y 25 años de edad, sin experiencia previa con el procedimiento, quienes fueron asignados aleatoriamente a los grupos experimentales
\end{abstract}

Situación experimental

El experimento se llevó a cabo bajo las condiciones y con los materiales descritos en el método general.

\title{
Tarea Experimental
}

Se empleó la tarea descrita en el método general.

\section{Procedimiento}

Los participantes fueron asignados a los grupos aleatoriamente. En la Tabla 1, se muestra el diseño experimental empleado. El entrenamiento fue igual para ambos grupos, en donde la plataforma invisible se ubicó entre los objetos guías A-B durante la primera fase de entrenamiento y entre los objetos guías C-B durante la segunda fase de entrenamiento. Se contrabalanceo la posición de la plataforma en la mitad de cada uno de los grupos. Ambas fases se conformaron de 12 ensayos de entrenamiento cada una. La fase de prueba se realizó a las 0 ó 24 h, dependiendo del grupo. Al entrar al cuarto experimental se les pidió a todos los participantes que se sentaran frente a la pantalla de la computadora el experimentador les leyó las instrucciones, se pulsó la barra espaciadora para poder continuar con la presentación de cada pantalla una vez que los participantes asentían entender la tarea y entonces el experimentador los dejaba solos en la habitación.

Los participantes realizaron un ensayo de familiarización con las flechas de navegación del teclado de la computadora, con la plataforma visible, pero sin los objetos que fungían como guías de referencia. Para continuar después de cada ensayo debían pulsar la barra espaciadora. El ensayo terminaba cuando los participantes encontraban la plataforma, recibiendo una retroalimentación en una pantalla que decía "Objetivo alcanzado", como se muestra en la Figura 1, o después de 60 s, recibiendo la retroalimentación que les decía "Objetivo no alcanzado". Posteriormente al ensayo de familiarización se presentaron los 24 ensayos de entrenamiento con la plataforma invisible. El programa informático registró la latencia de la respuesta de encontrar la plataforma y el tiempo de permanencia en cada cuadrante de la piscina circular. Finalmente en una fase de prueba los participantes tuvieron la oportunidad de "nadar" en la piscina por $60 \mathrm{~s}$, sin plataforma y con todos los objetos guías presentes. Durante esta fase se registró el tiempo que cada participante pasaba en cada cuadrante. En caso del grupo $0 \mathrm{~h}$ la prueba se aplicó inmediatamente después de la segunda fase, mientras que en el grupo de 24 h la prueba se aplicó 24 horas después. 
Tabla 1.

Diseño del Experimento 2

\begin{tabular}{lllll}
\hline Grupo & Fase1 & Fase2 & IR & Prueba \\
\hline $0 \mathrm{~h}$ & $\mathrm{~A}-\mathrm{B}+$ & $\mathrm{C}-\mathrm{D}+$ & $0 \mathrm{~h}$ & $60 \mathrm{~s}$ \\
$\mathrm{n}=8$ & $\mathrm{C}-\mathrm{D} \varnothing$ & $\mathrm{A}-\mathrm{B} \varnothing$ & & \\
& $(12)$ & $(12)$ & & \\
$24 \mathrm{~h}$ & $\mathrm{~A}-\mathrm{B}+$ & $\mathrm{C}-\mathrm{D}+$ & $24 \mathrm{~h}$ & $60 \mathrm{~s}$ \\
$\mathrm{n}=8$ & $\mathrm{C}-\mathrm{D} \varnothing$ & A-B $\varnothing$ & & \\
& $(12)$ & $(12)$ & & \\
\hline
\end{tabular}

Nota: donde los nombres de los grupos corresponden al momento de realizar la prueba, despúes de 0 horas o 24 horas ( 0 h y 24 h) con 8 participantes cada uno, A-B y C-D corresponden los cuadrantes que formaron los objetos ( $\mathrm{A}=$ pelota, $\mathrm{B}=\mathrm{cono}, \mathrm{C}=$ esfera y $\mathrm{D}=\mathrm{cubo}$ ) en donde se ubico la plataforma, $+=$ plataforma, $\varnothing=$ no plataforma y 60 s que duró el ensayo de prueba.

Resultados

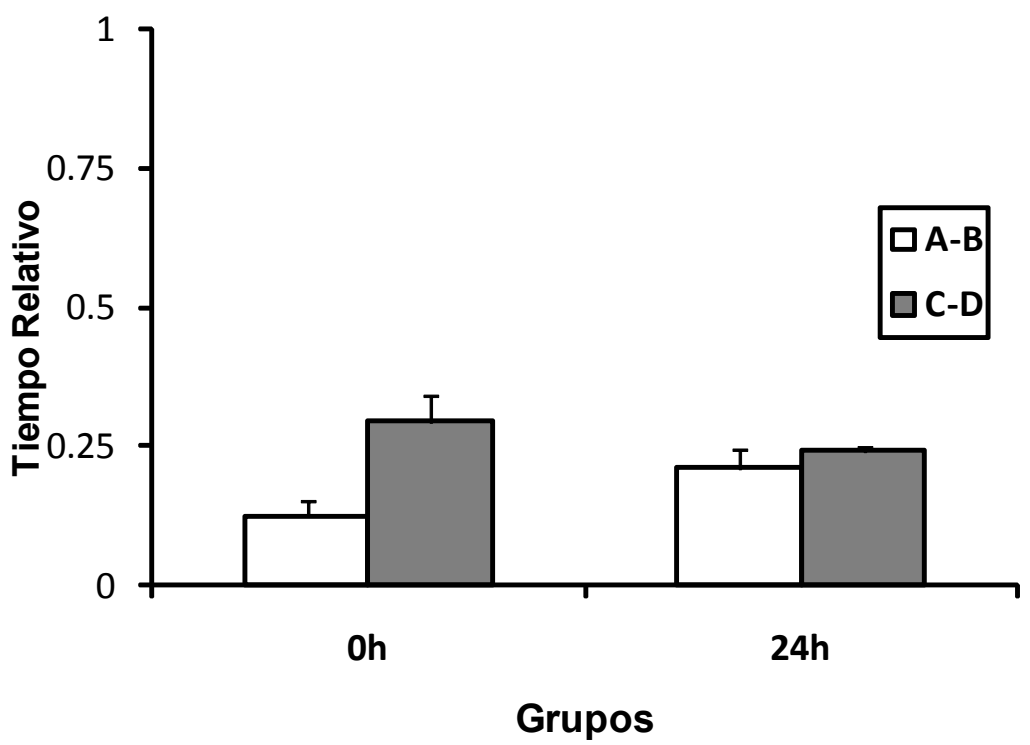

Figura 3. La figura muestra el tiempo relativo que pasaron los participantes en los cuadrantes en donde se ubicó la plataforma (A-B y C-D, durante la primera y segunda fase respectivamente) en los grupos 0 y 24 horas. 
Los resultados mostraron que todos los participantes aprendieron la tarea y disminuyeron su latencia para encontrar la plataforma de salida en la primera fase en donde se ubicó la plataforma en el cuadrante A-B y en el cambio de cuadrante C-D de la plataforma durante la segunda fase de entrenamiento Un ANOVA mixto de 2 grupos (0h y 24h) x 2 fases $(A B+, C D+)$, mostró diferencias significativas sólo entre las fases de adquisición e interferencia $(F(1,3)=4.14942, p<0.05)$. Lo que significa que todos los participantes cambiaron sus respuestas del cuadrante A-B durante la primera fase al cuadrante C-D en la segunda fase. Adicionalmente los participantes a los que se les presentó el ensayo de prueba a las 0 h, después de las dos fases de entrenamiento, pasaron más tiempo en el cuadrante donde fue ubicada la plataforma durante la segunda fase. Mientras que los participantes a los que se les presentó el ensayo de prueba después de $24 \mathrm{~h}$, del entrenamiento pasaron más tiempo en el cuadrante en donde estuvo ubicada la plataforma durante la primera fase de entrenamiento. La Figura 3 muestra un efecto de recencia en el grupo 0h ya que los participantes pasaron más tiempo (tiempo relativo) "nadando" en el cuadrante C-D, en el cual durante la fase 2 se ubicó la plataforma de salida. Por otro lado, en el grupo $24 \mathrm{~h}$ los participantes pasaron el mismo tiempo (tiempo relativo) "nadando" en ambos cuadrantes señalizados por los objetos (A-B y C-D), lo que demuestra una recuperación de la respuesta observada durante la primera fase de entrenamiento. Un ANOVA mixto de 2 condiciones (cuadrante $A B+y$ cuadrante $C D+$ ) $x 2$ grupos (test Oh y $24 \mathrm{~h}$ ) mostró una interacción $F(1,14)=6.72, p=<0$, y diferencias entre cuadrantes $p=<0.05$. Lo qué significa que los participantes del grupo Oh pasaron más tiempo en el cuadrante donde fue ubicada la plataforma durante la segunda fase, que en el cuadrante donde se ubicó durante la primera fase, mientras que los participantes del grupo $24 \mathrm{~h}$ pasaron igual tiempo en ambos cuadrantes durante el ensayo de prueba, lo que significa que los participantes después de un intervalo de retención regresan a buscar la plataforma en el cuadrante de la primera fase.

Estos resultados son acordes con la recuperación espontánea observada en estudios con aprendizaje causal (Alvarado, Jara, Vila \& Rosas, 2006) y en el condicionamiento instrumental con humanos (López, García \& Vila, 2010), ya que de forma similar a las preparaciones anteriores, los participantes recuperan la respuesta de nado en el cuadrante en el que se ubicó la plataforma durante la fase de adquisición en una prueba realizada después de un intervalo de tiempo. Por otro lado, la recuperación de la respuesta de "nado" observada en la primera fase en la tarea, ocurre de manera similar a la demostración con ratas en la piscina de Morris, (Prados, et al., 2003), cuando se entrena una fase de adquisición seguida de una fase de extinción. Así de manera general después de una fase de adquisición en la que todos los participantes aprenden la localización de la plataforma en un cuadrante y en una segunda fase de interferencia en la que aprenden una nueva localización de la plataforma en otro cuadrante, buscan la plataforma en una prueba inmediata en la última localización aprendida, sin embargo, en una prueba demorada buscan nuevamente en el primer cuadrante en el que se localizó la plataforma inicialmente. 


\section{Discusión}

Los resultados del Experimento 1 demuestran, que los participantes aprendieron a ubicar la plataforma invisible considerando los objetos que se ubicaron por fuera de la piscina, lo que implica, en primer lugar, que la tarea de nado virtual produce resultados similares a la preparación de nado en piscina de Morris con ratas (Morris, 1981) y a estudios con humanos que han empleado una piscina virtual (Sandstrom, et. al., 1998; Livingstone, \& Skelton, 2007). En segundo lugar, que los humanos aprendemos a ubicarnos espacialmente mediante la asociación de las señales espaciales que encontramos en el entorno.

Por otro lado, durante el ensayo de prueba en los grupos del Experimento 2, los participantes del grupo $0 \mathrm{~h}$ pasaron más tiempo en el cuadrante donde estuvo la plataforma durante la segunda fase, mientras que los participantes del grupo $48 \mathrm{~h}$ pasaron más tiempo en el cuadrante en donde se ubicó la plataforma durante la primera fase, éstos resultados son típicos del efecto de recuperación espontanea que se ha observado en una gran variedad de preparaciones tanto con animales no humanos como en animales humanos. Por lo tanto, es posible sugerir que la recuperación espontánea en aprendizaje espacial ocurre de manera similar que en animales entrenados en piscina de Morris (Prados, et al., 2003), y es también similar a lo observado con humanos en otras preparaciones como el aprendizaje verbal (Postman, Stark, \& Fraser,1968), el aprendizaje causal (Alvarado, et al., 2006) o el condicionamiento instrumental (López, et al., 2010)

Adicionalmente la ocurrencia de la recuperación espontánea, que es actualmente uno los efectos más robustos del aprendizaje asociativo (Rescorla, 2004), sugiere la posibilidad de que en el aprendizaje espacial, se asocien señales del espacio con la respuesta de encontrar un objetivo. Lo que es coherente con las ideas de Chamizo, $(2002,2003)$ y Pearce (2009) quienes aportan evidencia de un mecanismo asociativo en el aprendizaje espacial. Ya que al igual que en preparaciones de condicionamiento clásico como son el bloqueo, el desbloqueo y el ensombrecimiento, las claves espaciales compiten por ser las mejores predictoras de la respuesta, en éste caso, la que mejor señale la ubicación de la plataforma invisible. Así, la demostración de los fenómenos de aprendizaje asociativo como los antes mencionados, demuestra que las señales espaciales proporcionan información que compiten por ganar fuerza asociativa como lo predice el modelo de Rescorla y Wagner (1972) por ejemplo.

Por otro lado, la demostración de recuperación espontánea en aprendizaje espacial puede explicarse con las principales teorías de la recuperación de información como son: La teoría del desuso, originalmente propuesta por Thorndike (1911), en donde la información que no se usa por algún las representaciones en la memoria decaen. O la propuesta del desvanecimiento de la huella propuesta por Brown (1958) o la de consolidación proveniente del área de neurobiología, en donde el paso del tiempo es un requisito para que la información se consolide, con el riesgo que durante éste lapso de tiempo la fijación de la información queda vulnerable a otros estímulos 
(Mueller, \& Pilzecker, 1900). Así de acuerdo a las teorías anteriores parece que es la información que se aprende en un segundo momento la que sufre éstas afectaciones por lo que es posible entonces recuperar la información originalmente aprendida. Como es bien sabido, una teoría que goza de gran aceptación en el tema de recuperación espontánea es la Teoría de la interferencia (Bouton, 1993;1994) ya que el paso del tiempo es visto como un cambio de contexto, por lo que en el momento de la prueba es posible recuperara la información original, ya que es la segunda información la que requiere de señales contextuales para ser recuperada. Así con este punto de vista los resultados del segundo experimento pueden explicarse con la teoría de la interferencia de Bouton (1993;1994), en la cual la recuperación espontánea es un tipo de renovación en el cual el cambio de contexto producido por el paso del tiempo después de una fase de interferencia produce el resurgimiento de la respuesta aprendida originalmente durante la fase de adquisición.

Así de acuerdo con esta idea, para los participantes del grupo 24h ocurre un cambio de contexto debido al intervalo de $24 \mathrm{~h}$, lo que permite que se recupere la respuesta de "nado" de la primera fase de entrenamiento, mientras que en el grupo Oh no ocurre tal cambio y los participantes responden de acuerdo al entrenamiento de la segunda fase. Lo que sugiere que con el paso del tiempo los participantes recuerden mejor la ubicación de la plataforma al cambiar el contexto después de 24 h.

Por lo tanto los presentes resultados suponen una vía para comprender los mecanismos subyacentes al aprendizaje espacial y su relación con la recuperación de información, ya que éstas capacidades son esenciales en el comportamiento de numerosas especies para su supervivencia, desde los animales en conductas como búsqueda de alimento (Cabrera, 2009) hasta las implicaciones terapéuticas en problemas de deterioro de memoria.

\section{Referencias}

Alvarado, A., Jara, E., Vila, J., \& Rosas, J., M. (2006). Time and order effects in causal learning. Learning and Motivation. 37, 324-345. Available via: http://dx.doi. org/10.1016/j.Imot.2005.11.001

Bouton, M., E. (1993). Context, time and memory retrieval in the interference paradigms of Pavlovian learning. Psychological Bulletin, 114, 80-99. Available via: http://dx.doi.org/10.1037//0033-2909.114.1.80

Bouton, M., E. (1994). Conditioning, remembering and forgetting. Journal of Experimental Psychology: Animal Behavior Processes, 20(3), 219-231. Available via: http://dx.doi.org/10.1037//0097-7403.20.3.219

Brown, J. (1958) Some test of the decay theory of immediate memory. Quarterly Journal of Experimental Psychology, 10, 10-21. Available via: http://dx.doi. org/10.1080/17470215808416249 
Cabrera, F. (2009). Búsqueda de alimento en hámsteres dorados (mesocricetus auratus): el efecto de la distancia entre múltiples fuentes de alimento. Universitas Psychologica, 7(1), 125-138.

Chamizo, V., D. (2002). Spatial Learning: Conditions and Basic Effects. Psicológica, 23, 33-57.

Chamizo, V., D. (2003). Acquisition of knowledge about spatial location: Assessing the generality of the mechanism of learning. Quarterly Journal of Experimental Psychology, 56b, 102-113. Available via: Available via: http://dx.doi. org/10.1080/02724990244000205

Chamizo, V., D., Azar-Casanova, J., A., \& Artigas, A., A. (2003) Human overshadowing in a virtual pool: Simple guidance is a good competitor against locale learning. Learning and Motivation, 34, 262-281. Available via: http://dx.doi.org/10.1016/ S0023-9690(03)00020-1

Hull, C., L. (1943). Principles of behavior. New York: Appleton-Century-Crofts. Available via: http://dx.doi.org/10.1097/00005053-194504000-00026

López-Romero, L., J., García-Barraza, R., \& Vila J. (2010). Spontaneous recovery does not appear as a recency-to-primacy shift as a function of the retention interval value in human instrumental learning. Behavioral Proccesses. 84, 617621. Available via: http://dx.doi.org/10.1016/j.beproc.2010.01.007

Morris, G., M. (1981). Spatial localization does not require the presence of local cue. Learning and Motivation, 12, 239-260. Available via: http://dx.doi. org/10.1016/0023-9690(81)90020-5

Mueller, G.E., \& Pilzecker, (1900) Experimentelle beitrage zur lehre von gedachtnis. Zeitschrift fur psychologie, 1, 1-30.

O'Keefe, J., \& Nadel, L. (1978). The hippocampus as a cognitive map. Oxford: Oxford University Press. Available via: http://dx.doi.org/10.1002/hipo.450010303

Pavlov, I., P. (1927). Conditioned reflexes. Oxford: Oxford University Press. Available via: http://dx.doi.org/10.1037/11081-021

Prados, J. (1999). Latent Inhibition in a Navigation Task: Evidence for the Use of Associative processes in spatial memory. Psicológica, 20, 151-162.

Prados, J. (2000). Effects of varying the amount of preexposure to spatial cues on a subsequent navigation task. Quarterly Journal of Experimental Psychology, 53b, 139-148. Available via: http://dx.doi.org/10.1080/027249900392931

Prados, J., Chamizo, V., D., \& Mackintosh, N., J. (1999). Latent inhibition and perceptual learning in a swimming-pool navigation task. Journal of Experimental Psychology:Animal Behavior Processes, 25, 37-44. Available via: http://dx.doi. org/10.1037//0097-7403.25.1.37

Prados, J., Manteiga, R., D., \& Sansa, J. (2003). Recovery effects after extinction in the Morris swimming pool navigation task. Learning \& Behavior, 31, 299-304. Available via: http://dx.doi.org/10.3758/BF03195991

Postman, L., Stark, K., \& Fraser, J. (1968) Temporal changes in interference. Journal 
of Verbal Learning and Verbal Behavior, 7, 672-694. Available via: http://dx.doi. org/10.1016/S0022-5371(68)80124-0

Restle, F. (1957). Discrimination of cues in mazes: a resolution of the 'place-vsresponse' question. Psychological Review, 65, 77-91. Available via: http://dx.doi. org/10.1037/h0040678

Rescorla, R. (2004). ). Spontaneous recovery varies inversely with the training- extinction interval. Learning \& Behavior, 32, 401-408. Available via: http://dx.doi. org/10.3758/BF03196037

Sandstrom, N., J., Kauffman, J., \& Huettel, S., A. (1998). Males and females use different distal cues in a virtual environment navigation task. Cognitive Brain Research, 6, 351 - 360. Available via: http://dx.doi.org/10.1016/S0926-6410(98)00002-0

Livingstone, S., A., \& Skelton, R., W. (2007). Virtual environment navigation task assessment of cognitive deficits in individuals with brain injury. Behavioural Brain Research, 185, 21-31. Available via: http://dx.doi.org/10.1016/j.bbr.2007.07.015

Shettlewoorth, S., J. (2010). Cognition, evolution and behavior. New York: Oxford University Press.

Thorndike, E. L. (1911). Animal intelligence: Experimental studies. New York, Macmilan.

Tolman, E., C. (1948). Cognitive maps in rats and men. Psychological Review, 55, 189-208. Available via: http://dx.doi.org/10.1037/h00616260 